\title{
Mathematical modelling of Chlamydia
}

\author{
D. P. Wilson*
}

(Received 8 August 2003; revised 31 March 2004)

\begin{abstract}
Chlamydiae are intracellular bacterial parasites that develop and multiply within mucosal cells of humans and animals and amongst the most common sexually transmitted disease worldwide. If left untreated, infection with Chlamydia can lead to serious outcomes such as infertility, ectopic pregnancy and blinding trachoma due to tissue scarring. There are currently no vaccines available for these major diseases. Microbiological research on Chlamydia is advancing rapidly and recently mathematical models of the bacteria have been developed to assist understanding of the complex chlamydial developmental cycle. Here, we extend the current description of the chlamydial developmental cycle and review the models developed and their predictions about chlamydial pathogenesis. The computational techniques employed by these applications are outlined.
\end{abstract}

${ }^{*}$ QUT, Brisbane, Australia. mailto:dp.wilson@qut.edu.au

See http://anziamj.austms.org.au/V45/CTAC2003/Wils for this article, (C) Austral. Mathematical Soc. 2004. Published April 13, updated April 14, 2004. ISSN 1446-8735 


\section{Contents}

1 Introduction

C202

2 Basic overview model

C203

3 Modelling humoral immunity: $\mu / \kappa_{1}$

C205

4 Modelling cell-mediated immunity: $\gamma / \kappa_{2}$

C206

5 Within-host modelling: $P$

C207

5.1 Developmental cycle model . . . . . . . . . . . . . C208

5.2 Application to analysis of gene expression . . . . . . . C209

6 Discussion

C211

References

C212

\section{Introduction}

Chlamydiae are bacterial pathogens and responsible for many serious conditions including blindness and pelvic inflammation leading to infertility in women. Disease due to invading Chlamydia commences as chlamydial particles infect epithelial cells of mucosal linings. However, the immune system responds via two mechanisms, namely, humoral immunity and cell-mediated immunity. In this paper, we develop a mathematical modelling framework for chlamydial infection and review the models developed to date.

Free extracellular chlamydial particles are susceptible to humoral immunity. Humoral immunity refers to immune protection mediated by B lymphocytes and provided by circulating antibodies against foreign pathogens introduced to the body. Antibodies neutralize a chlamydial particle by attaching to its surface binding sites, blocking the pathogen from attaching to 
target cell receptors and so cell entry is inhibited. Additionally, antibodies are involved in signalling macrophage cells of the immune system to engulf the bound pathogen. Chlamydia are intracellular in nature for reproduction. Thus, preventing entrance to host cells would be the terminal event for Chlamydia. However, humoral immunity is not entirely effective and so host cells are infected with Chlamydia.

It has been suggested that cell-mediated immunity, not humoral immunity, plays the dominant role in protective immunity against Chlamydia [12]. The importance of cell-mediated immunity to chlamydial infection has been emphasized in a number of studies [3, 7]. Cell-mediated immunity acts as T lymphocytes secrete proteins called cytokines to act directly or indirectly against the pathogen and also stimulate cytotoxic T cells. Cytotoxic T cells play an important role in protecting the host against chlamydial infection by clearing infected cells.

\section{Basic overview model}

We let $C(t)$ be the concentration of free extracellular chlamydial particles, $E(t)$ be the number of uninfected mucosal epithelial cells (main host cell for Chlamydia), and $I(t)$ be the number of Chlamydia-infected epithelial cells at time $t$, and if we simplify the cellular dynamics by modelling interactive processes by parameters, then a simple mathematical description of the change in the interacting 'species' is

$$
\begin{aligned}
\dot{C} & =P \kappa_{2} I(t)-\mu C(t)-\kappa_{1} C(t) E(t), \\
\dot{E} & =P_{E}-\delta_{E} E(t)-\kappa_{1} C(t) E(t), \\
\dot{I} & =\kappa_{1} C(t) E(t)-\gamma I(t)-\kappa_{2} I(t) .
\end{aligned}
$$

Here, $P$ is the number of chlamydial particles released from infected cells at a rate of $\kappa_{2}, \mu$ is the rate of clearance by macrophages, $\kappa_{1}$ is the rate of epithelial cell infection, which may be reduced by antibodies. Epithelial 
cells are produced at a rate $P_{E}$ and $\delta_{E}$ is the rate of epithelial cell natural death. We let $\gamma$ be the rate of clearance of infected cells due to cell-mediated immunity. There are a number of factors influencing the pathogenesis of chlamydial infection. We can see this by examining steady states and the basic reproduction ratio.

Our model system of equations (1-3) has a trivial steady state

$$
\bar{C}=0, \quad \bar{E}=P_{E} / \delta_{E}, \quad \bar{I}=0,
$$

and a non-trivial steady state

$$
\begin{aligned}
\bar{C} & =\frac{P_{E}\left[(P-1) \kappa_{2}-\gamma\right]}{\mu\left(\gamma+\kappa_{2}\right)}-\frac{\delta_{E}}{\kappa_{1}}, \\
\bar{E} & =\frac{\mu\left(\gamma+\kappa_{2}\right)}{\kappa_{1}\left[(P-1) \kappa_{2}-\gamma\right]}, \\
\bar{I} & =\frac{P_{E}}{\gamma+\kappa_{2}}-\frac{\delta_{E} \mu}{\kappa_{1}\left[(P-1) \kappa_{2}-\gamma\right]},
\end{aligned}
$$

corresponding to clearance of infection and active disease respectively.

The basic reproduction ratio is a fundamental concept in micro-epidemiology [4] and is a criterion relating parameter values to determine whether the system will have an increasing or decreasing solution. The basic reproduction ratio is denoted as $R_{0}$ and is defined as the expected number of secondary cases produced, in a completely susceptible population, by one typical infected individual/cell during its entire period of infectiousness. The basic reproduction ratio is evaluated when abundance of uninfected cells is at pre-infection level. We obtain the following simplified expression for the basic reproductive ratio, namely,

$$
R_{0}=\frac{P}{\left(1+\gamma / \kappa_{2}\right)\left(1+\mu /\left(\kappa_{1} E_{0}\right)\right)},
$$

where $E_{0}=P_{E} / \delta_{E}$. The trivial steady state is stable when $R_{0}<1$ and the non-trivial steady state is stable when $R_{0}>1$. We note that increased ability to clear infection will be obtained if 
TABLE 1:

\begin{tabular}{ll}
\hline Parameter & Value \\
\hline$P:$ burst size per infected cell & $200-500$ \\
$\kappa_{1}:$ rate of cell infection & $0.02 \mathrm{~mm}^{3} /$ day $/$ cell \\
$\kappa_{2}:$ rate infected cells burst & $0.33-0.6$ days $^{-1}$ \\
$P_{E}:$ rate of production of mucosal epithelial cells & 40 cells $/ \mathrm{mm}^{3} /$ day \\
$\delta_{E}:$ rate of natural death of epithelial cells & 2 days \\
$\gamma:$ effectiveness of cell-mediated immunity & $2-10$ days $^{-1}$ \\
$\mu:$ effectiveness of humoral immunity & $2-10$ days $^{-1}$ \\
\hline
\end{tabular}

1. macrophage engulfment of chlamydial particles occurs prior to host cell infection $\left(\mu / \kappa_{1}\right)$,

2. the cytotoxic immune response clears infected cells prior to lysis of the infected cell $\left(\gamma / \kappa_{2}\right)$, or

3. the number of infectious chlamydial particles released by an infected cell is reduced $(P)$.

The relative importance of these factors can be seen by estimates in parameter values in Table 1 . In the following sections we review the mathematical models developed that investigate each of these aspects and indicate how computational techniques have been used in these applications.

\section{Modelling humoral immunity: $\mu / \kappa_{1}$}

Wilson and McElwain modelled humoral immunity against chlamydial challenge by tracking the antibody and host cell receptor aggregation over chlamydial particles [10]. Tracking the distribution of antibodies aggregating on chlamydial particles is important because of the large number of binding sites and high antibody concentration required for neutralization. A deeper 
understanding of chlamydial neutralization would be of benefit in the development of a vaccine.

Steric hindrance greatly influences the number of available sites that may be bound, reducing relative occupancy well below 100\% [6]. To determine the effective neutralization and substantiate neutralization theory the model was fitted to a discrete time-course for the antibody concentration and the percentage of mucosal cells infected with time. Firstly, a continuous timecourse for the antibody concentration was obtained by fitting cubic splines to experimental data using the standard cubic splines textbook algorithm. Secondly, the model was fitted to the percentage of infected cell data. This was achieved by minimizing the objective error function defined to be the sum of squares of differences of the number of infected cells and model predicted number of infected cells over all experimental time points. The model prediction is a function of four unknown parameters. The method of steepest decent was used to obtain the parameters of best fit. Here, first order approximations were used for the gradient. Additionally, the number of infected cells predicted by the model was determined by the solution of a system of differential equations and was solved with a simple finite difference algorithm.

\section{Modelling cell-mediated immunity: $\gamma / \kappa_{2}$}

It is assumed that the magnitude of the cell-mediated immune response is proportional to the number of replicating forms of chlamydial particles within a host cell. Chlamydiae have a complex replicative cycle complicating the tracking of intracellular chlamydial particles. Additionally, the response will change as the number of cytotoxic $\mathrm{T}$ cells increases with time due to immune responsiveness.

Wilson, Timms and McElwain modelled the inter-conversion between infectious and replicating chlamydial particles to track the number of particles within a host cell with time over the developmental cycle [11]. Cellular 
dynamics were modelled exterior to the within-host model and immune effectiveness was studied. An age-structured PDE was developed to account for the different immune response over an infected cell's lifetime. The agestructured component could be solved in closed analytic form (as an integral solution). However, the solution to the entire system was performed numerically and finite difference algorithms employed. To manage the integral components, Simpson's quadrature rule was used. Conclusions were made about the influence of each process within the system, including the effects of an important persistent form of chlamydial infection. This modelling has lead to further investigations of within-host dynamics.

\section{Within-host modelling: $P$}

Within-host mathematical modelling of infectious diseases has not been utilised extensively. In this section we establish a framework for within-host modelling of Chlamydia's unique developmental cycle. The chlamydial life cycle is initiated when an infectious, extracellular transmission cell, termed the elementary body (EB), attaches to a susceptible host cell. The host cell internalizes the EB, where, the EB undergoes morphological changes, reorganizing to the larger reticulate body (RB), the replicative form of Chlamydia. At this stage, the RBs multiply 200-300 fold by binary fission. At 20-25 hours after infection, infectious EBs appear as the EB to RB conversion process is reversed. Some RBs continue to divide whilst others reorganise into EBs, resulting in asynchronous growth, until the host cell cannot support the multiplication any longer. The host cell bursts, releasing the EBs, thereby initiating new cycles of infection. Depending on the strain of Chlamydia, the developmental cycle takes between 40 and 72 hours. The metamorphosis of EB to RB includes passage via an intermediate body (IB). Although it is usually thought that one RB will condense to one EB, often more than one EB may be formed as IBs have also been seen in the act of division [8]. Thus, we distinguish between forward and backward EB to RB intermediate bodies. 
Electron microscopy has also shown that in an intervening period when many cells appear not to be infected there are sometimes chlamydiae present. However, these chlamydiae consist of structurally abnormal RBs. It has been found that incomplete, persistent chlamydial infection can be induced from cell-mediated immunity against chlamydial infection [2]. Additionally, although many studies have shown efficient inhibition of infection with antibiotics, elimination of chlamydiae is not necessarily the result of prolonged treatment. Instead, chlamydiae are often temporarily arrested in a persistent state, stressed out of the RB form and suppressed in replication [5]. Since antibiotics attack chlamydiae differently to the cell-mediated response, the persistent particles induced by the antimicrobial agents will be different. However, it is difficult to distinguish between the two spectrums of abnormal particles. Here, we refer to a chlamydial particle that has been induced into this abnormal state by either pathway as an aberrant body (AB).

\subsection{Developmental cycle model}

We let $R_{B}(t)$ and $E_{B}(t)$ be the numbers of RBs and EBs in the host cell at time $t$ and $I_{B 0}(r, t)$ and $I_{B}(r, t)$ be the number of IBs that are in the process of transforming from an EB to a $\mathrm{RB}$, and from a $\mathrm{RB}$ to an EB respectively at time $t$, where $r$ is the degree of maturation along the transformation process since there is a spectrum of IBs between the EB and RB forms (there is also a spectrum of ABs). Here, $r_{0} \leq r \leq r_{1}$, where $r_{0}$ and $r_{1}$ denote an EB and RB respectively. We also let $A_{B}(s, t)$ be the number of ABs at time $t$ with degree of aberration measured by the parameter $s$, where $s_{0} \leq s \leq s_{1}$ and $s=s_{0}$ denotes a normal RB. Then, the progression through the developmental cycle can be expressed mathematically by the following general system of 
equations,

$$
\begin{aligned}
\dot{I}_{B 0}= & -\frac{\partial}{\partial r}\left\{k_{f}(r, t) I_{B 0}(r, t)\right\}, \\
\dot{R}_{B}= & k_{f}\left(r_{1}, t\right) I_{B 0}\left(r_{1}, t\right)+\left[\rho\left(s_{0}, t\right)-c(t)-k_{a f}\left(s_{0}, t\right)\right] R_{B}(t) \\
& +k_{a r}\left(s_{0}, t\right) A_{B}\left(s_{0}, t\right), \\
\dot{A}_{B}= & -\frac{\partial}{\partial s}\left\{\left(k_{a f}(s, t)-k_{a r}(s, t)\right) A_{B}(s, t)\right\}+\rho(s, t) A_{B}(s, t), \\
\dot{I}_{B}= & \frac{\partial}{\partial r}\left\{k_{r}(r, t) I_{B}(r, t)\right\} \\
\dot{E}_{B}= & k_{r}\left(r_{0}, t\right) I_{B}\left(r_{0}, t\right) .
\end{aligned}
$$

Here, $k_{f}$ is the rate of maturing of an IB towards the RB and $k_{r}$ is the rate of reverse maturation of an IB towards an EB. The rate an AB progresses in its degree of aberration to/from an RB is given by $k_{a}=k_{a f}-k_{a r}$ and depends on the concentration of antimicrobial agent in the system. We let $c$ be the rate of RB commitment to transform to EBs and $\rho$ be the rate of replication of RBs and aberrant RBs (ABs). We set the initial conditions $I_{B 0}\left(r_{0}, 0\right)=1, I_{B 0}(r \neq$ $\left.r_{0}, 0\right)=R_{B}(0)=A_{B}(s, 0)=I_{B}(r, 0)=E_{B}(0)=0$, and the boundary conditions $\dot{I_{B 0}}\left(r_{0}, t\right)=0, \dot{A_{B}}\left(s_{0}, t\right)=k_{a f}\left(s_{0}, t\right) R_{B}(t), \dot{I_{B}}\left(r_{1}, t\right)=c(t) R_{B}(t)$. A finite difference algorithm was employed, using centred-difference formulas for derivatives, to solve the system of equations (5-9). Figure 1 illustrates the numerical solution to the above system under two conditions, namely, normal chlamydial development (persistent ABs are not induced) and persistent chlamydial development. The form of solutions and resultant values are typical of what is seen in the literature (see, for example, Beagley and Timms [1]).

\subsection{Application to analysis of gene expression}

Chlamydial growth necessitates the ordered and differential expression of the right genes at the right time for the morphological and functional events in 

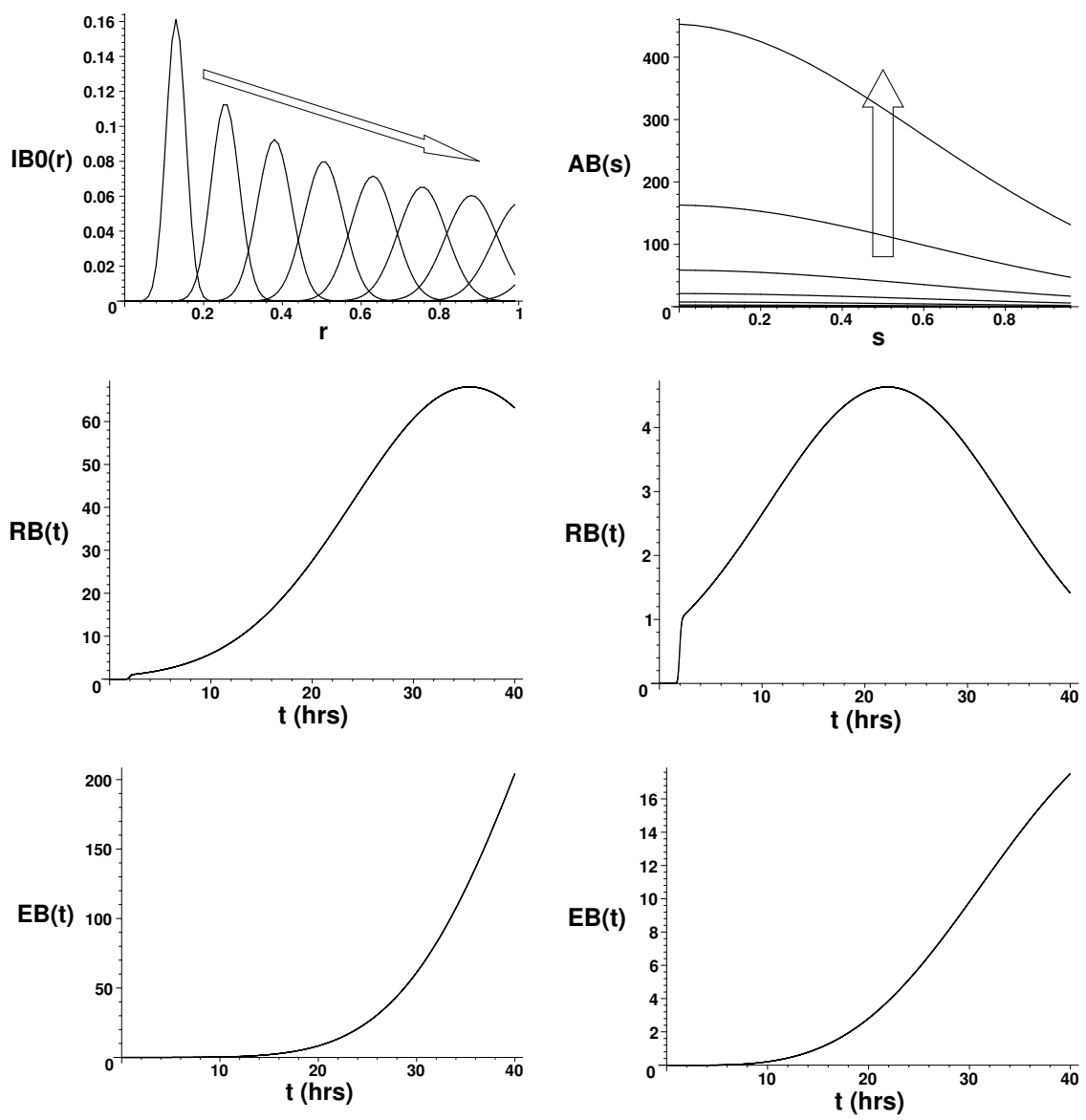

Figure 1: Left Panel: (Normal growth) Numerical solutions illustrate: (a) the development of $I_{B 0}$ for various times where the arrow indicates how the solution changes with time; (b) the time-courses of the number of RBs and (c) EBs per host cell. Right Panel: (Persistent development) Numerical solutions illustrate: (a) the development of $A_{B}$ for various times where the arrow indicates how the persistence solution changes with time; (b) the time-courses of the number of RBs and (c) EBs per host cell. Here, parameter values used for simulations shown in the top and bottom panel are $k_{f}=k_{f}=0.5, \rho(s)=0.267\left(s_{1}-s\right) /\left(s_{1}-s_{0}\right) \mathrm{hrs}^{-1}, c=0.0075 t \mathrm{hrs}^{-1}$. Then, in the normal growth simulation, $k_{a f}=k_{a r}=0$ and in the persistence simulation, $k_{a f}=0.1, k_{a r}=0$. 
the developmental cycle to occur. Relatively little is known about the gene regulation. However, enormous amounts of gene expression data is emerging from microbiologists and mathematical models such as the one presented in the previous section will be invaluable in standardizing the gene expression and making prediction of crucial signalling and triggers of Chlamydia. This would reveal clearly defined targets for microbiologists in drug and vaccine development. An example of the application of micro-array technology has been done by Wilson, Mathews, Wan and McElwain [9]. A constitutive gene's transcript levels were quantitatively measured, and a mathematical model used to standardize for the number of chlamydial particles. The model was used to predict the number of each type of chlamydial particle within a host cell and then estimate the RB doubling time and the maximum number of chlamydial particles per inclusion. The estimates were based on the statistical ideas of profile likelihoods. The algorithm required simulations be performed over the entire realistic parameter space. A fourth order Runge-Kutta method was used to solve the model system of coupled first order initial value equations. A standard steepest decent algorithm was used to obtain a search direction in parameter space for a better sequence of parameters (according to sum of squared residuals weighted by the variance of experimental data) until appropriate convergence of parameters was achieved (parameter values similar to 8 digits for subsequent iterations).

\section{Discussion}

We presented a basic overview of the avenues available for fighting chlamydial infection and described mathematical models developed to investigate humoral immunity, cell-mediated immunity and within-host dynamics. Perhaps the most promising area for future mathematical modelling of Chlamydia is in the application of emerging micro-array technology that measures gene expression. There is evidence to suggest that many antibiotics induce chronic persistent Chlamydia. The detailed modelling of the cause and con- 
sequences of ABs, including the adverse effects of antibiotic treatment is also an area awaiting investigation. Most of the severe sequelae of chlamydial infection occur as a result of long-term inflammation leading to fibrosis and scarring. However, the precise mechanisms by which scarring occurs is unknown. Scarring of the mucosal lining, and the cellular dynamics giving rise to inflammation are also topics in which insight can be gained though the use of mathematical modelling. Mathematical modelling has enabled much insight to be gained into many biological phenomena. Disease and other problems related to chlamydial infection is common around the world and an interdisciplinary approach, including mathematical modelling with the framework established here, is required if the long-term goal of a cure is to eventuate.

\section{References}

[1] K. W. Beagley, and P. Timms, Chlamydia trachomatis infection: incidence, health costs and prospects for vaccine development, J. of Reproductive Immunology. 48:47-68, 2000. C209

[2] W. L. Beatty, G. I. Byrne and R. P. Morrison. Morphologic and antigenic characterization of interferon $\gamma$-mediated persistent Chlamydia trachomatis infection in vitro. Proc. Natl. Acad. Sci., 90:3998-4002, 1993. C208

[3] T. K. Cain, and R. G. Rank. Local Th1-Like Responses Are Induced by Intravaginal Infection of Mice with the Mouse Pneumonitis Biovar of Chlamydia trachomatis. Infect. and Immun., 63:1784-1789, 1995. C203

[4] O. Diekmann, J. A. P. Heesterbeek and J. A. J. Metz. On the definition and the computation of the basic reproduction ratio $\mathrm{R} 0$ in 
models for infectious diseases in heterogeneous populations. J. Math. Biol., 28:365-382, 1990. C204

[5] U. Dreses-Werrringloer, I. Padubrin, H. Zeidler and L. Kohler. Effects of Azithromycin and Rifampin on Chlamydia trachomatis Infection In Vitro, Antimicrob. Agents Chemother., 45:3001-3008, 2001. C208

[6] P. J. Klasse and Q. J. Sattentau. Occupancy and mechanism in antibody-mediated neutralization of animal viruses. J. Gen. Virol. 83:2091-2108, 2002. C206

[7] D. M. Magee, D. M. Williams, J. G. Smith, C. A. Bleicker, B. G. Grubbs, J. Schacter and R. G. Rank. Role of CD8 T Cells in Primary Chlamydia Infection, Infect. Immun., 63:516-521, 1995. C203

[8] M. E. Ward. The chlamydial developmental cycle in pictures. http://www.chlamydiae.com/docs/biology/biol_devcycle.htm C207

[9] D. P. Wilson, S. Mathews, C. Wan, D. L. S. McElwain. Use of a quantitative gene expression assay based on micro-array techniques and a mathematical model for the investigation of chlamydial generation time and inclusion size, Bulletin of Mathematical Biology, Volume 66, Issue 3, May 2004, Pages 523-537. C211

[10] D. P. Wilson, D. L. S. McElwain. A model of neutralization of Chlamydia trachomatis based on antibody and host cell aggregation on the elementary body surface, Journal of Theoretical Biology, Volume 226, Issue 3, February 2004, Pages 321-330. C205

[11] D. P. Wilson, P. Timms, D. L. S. McElwain. A mathematical model for the investigation of the Th1 immune response to Chlamydia trachomatis. Math. Biosci., 182:27-44, 2003. C206 
[12] X. Yang and R. C. Brunham. T lymphocyte immunity in host defence against Chlamydia trachomatis and its implication for vaccine development, Canadian J. Infect. Dis., 9:99-108, 1998. C203 